\title{
Joint Discussion 10: 3D views on cool stellar atmospheres - theory meets observation
}

\section{Organizing committee:}

Carlos Allende Prieto (UK), Martin Asplund (Germany), Mats Carlsson (Norway), Márcio Catelan (Chile), Kwing Lam Chan (China), Dainis Dravins (Sweden), Hans-G. Ludwig (Chair, France), K.N. Nagendra (India), Åke Nordlund (Denmark), Natalia Shchukina (Ukraine), T. Sivarani (USA), and Matthias Steffen (Germany)

\section{Preface}

Much of what we know about the chemical composition of the Universe actually stems from the chemical composition of stars, which is often deciphered from the spectra emerging from their atmospheres. Cool, low-mass and long-living stars allow to study the evolution of the Universe's chemistry from a time shortly after the big bang until today. The observation and interpretation of stellar spectra is a classical field in astronomy but is still undergoing vivid developments. The enormous increase in available computational resources opened-up possibilities which led to a revolution in the degree of realism to which modelers can mimic Nature. High-resolution, high-stability, high-efficiency spectrographs are now routinely providing stellar spectra whose full information content can only be exploited if a very much refined description of a stellar atmosphere is at hand.

This situation motivated Commission 36 Theory of Stellar Atmospheres to organize an exchange of latest views on the modelling of atmospheres of cool stars, and their inherent complexities related to multi-dimensional hydrodynamics and magnetic fields. The idea materialized in the form of this Joint Discussion. Being a Joint Discussion ample time could be allocated to discussions, and indeed many lively disputes revolved around the diverse topics presented by the speakers and illustrated in posters. It is fair to say that they touched upon practically all aspects of stellar atmospheres - theoretical as well as observational with emphasis on their multi-dimensional nature.

Proceedings of the Joint Discussion were published in full in a dedicated volume of the Memorie della Società Astronomica Italiana. (Vol. 80, no. 3, 2009). The reprinted abstracts in this volume are intended to summarize the vivid exchange of views; they hopefully serve as a happy reminder to the participants of the 3D Views on Cool Stellar Atmospheres: Theory Meets Observation, and provide some flavor of the event to those who have missed it.

We wish to thank the IAU for promoting this Joint Discussion. We are grateful to all the participants who have made it a lively meeting, and in particular to the colleagues who chaired the different sessions. Special thanks are due to the Sociedade Astronômica Brasileira who took care of the organization of the IAU General Assembly and welcomed us at Rio de Janeiro.

K. N. Nagendra, P. Bonifacio, H.-G. Ludwig, editors 


\title{
Hydrodynamics and radiative transfer of 3D model atmospheres: current status, limitations, and how to make headway
}

\author{
M. Carlsson ${ }^{1,2}$ \\ ${ }^{1}$ Institute of Theoretical Astrophysics, University of Oslo, P.O. Box 1029 Blindern, \\ N-0315 Oslo, Norway \\ ${ }^{2}$ Center of Mathematics for Applications, University of Oslo, P.O. Box 1052 Blindern, \\ N-0316 Oslo, Norway
}

\begin{abstract}
D MHD models are important tools for advancing our understanding of stellar atmospheres. A major computational challenge is the treatment of radiative transfer; both to get a realistic treatment of the energy transfer in the 3D modelling and for the diagnostic problem of calculating the emergent spectrum in more detail from such models. The current status, limitations and future directions of 3D MHD atmospheric modelling and the treatment of radiative transfer are here discussed.
\end{abstract}

\section{Highest-resolution spectroscopy}

\author{
D. Dravins
}

Lund Observatory, Box 43, SE-22100 Lund, Sweden

\begin{abstract}
D models of stellar atmospheres predict spectral-line shapes with asymmetries and wavelength shifts, but the confrontation with observations is limited by blends, lack of suitable lines, imprecise laboratory wavelengths, and instrumental imperfections. Limits can be pushed by averaging many similar lines, thus averaging small random blends and wavelength errors. In non-solar cases, any detailed verification of 3-D hydrodynamics requires spectra of resolutions $\mathrm{R}=\lambda / \Delta \lambda \approx 300,000$, soon to become available. An issue is the optical interface of high-resolution spectrometers to [very] large telescopes with their [very] large image scales, possibly requiring adaptive optics. The next observational frontier may be spectroscopy across spatially resolved stellar disks, utilizing optical interferometers and extremely large telescopes.
\end{abstract}

\section{Granulation across the HR diagram}

I. Ramírez ${ }^{1}$, C. Allende Prieto ${ }^{2}$, D. L. Lambert ${ }^{3}$, L. Koesterke ${ }^{3,4}$ and M. Asplund ${ }^{1}$

${ }^{1}$ Max Planck Institute for Astrophysics, Postfach 1317, 85741 Garching, Germany

${ }^{2}$ Mullard Space Science Laboratory, University College London, UK

${ }^{3}$ McDonald Observatory and Dept. of Astronomy, University of Texas at Austin, USA

${ }^{4}$ Texas Advanced Computing Center, University of Texas, USA

Abstract. We have obtained ultra-high quality spectra $(R=180,000 ; S / N>300)$ with unprecedented wavelength coverage (4400 to $7400 \AA$ ) for a number of stars covering most of the HR diagram in order to test the predictions of models of stellar surface convection. Line bisectors and core wavelength shifts are both measured and modeled, allowing us to validate and/or reveal the limitations of state-of-the-art hydrodynamic model atmospheres of different stellar parameters. We show the status of our project and preliminary results.

\section{Accounting for convective blue-shifts in the determination of absolute stellar radial velocities}

C. Allende Prieto ${ }^{1}$, L. Koesterke ${ }^{2}$, I. Ramírez ${ }^{3}$, H.-G Ludwig $^{4}$ and M. Asplund ${ }^{3}$ 
${ }^{1}$ Mullard Space Science Laboratory, University College London, UK

${ }^{2}$ Texas Advanced Computing Center, The University of Texas at Austin, USA

${ }^{3}$ Max Plank Institute for Astrophysics, Garching, Germany

${ }^{4}$ CIFIST, GEPI, Observatoire de Paris, CNRS, Université Paris Diderot, France

Abstract. For late-type non-active stars, gravitational redshifts and convective blueshifts are the main source of biases in the determination of radial velocities. If ignored, these effects can introduce systematic errors of the order of $\sim 0.5 \mathrm{~km} \mathrm{~s}^{-1}$. We demonstrate that threedimensional hydrodynamical simulations of solar surface convection can be used to predict the convective blue-shifts of weak spectral lines in solar-like stars to $\sim 0.070 \mathrm{~km} \mathrm{~s}^{-1}$. Using accurate trigonometric parallaxes and stellar evolution models, the gravitational redshifts can be constrained with a similar uncertainty, leading to absolute radial velocities accurate to $\sim 0.1$ $\mathrm{km} \mathrm{s}^{-1}$.

\title{
$3 \mathrm{D}$ radiative transfer with continuum and line scattering in low arbitrary velocity fields
}

\author{
A. M. Seelmann and P. H. Hausschildt \\ Hamburger Sternwarte, Gojenbergsweg 112, 21029 Hamburg, Germany
}

\begin{abstract}
With the increasing computer power of modern supercomputers, full 3D radiative transfer calculations with scattering is becoming more and more feasible. The PHOENIX/3D code is a first step towards realistic 3D model atmospheres with scattering in the line and the continuum. Numerous 3D hydro-dynamical codes exist, for example the FLASH or the $\mathrm{CO}^{5} \mathrm{BOLD}$, which can calculate 3D hydro-dynamical structures for, e.g., the Sun. This work concentrates on bringing the two approaches together in the sense of doing realistic, timeindependent, radiative transfer with line scattering under considerations of the intrisitc velocity fields in a snapshot of the 3D hydro structure.
\end{abstract}

\section{Projection methods for line radiative transfer in spherical media}

L. S. Anusha and K. N. Nagendra

Indian Institute of Astrophysics, Koramangala 2nd Block, Bangalore 560 034, India

\begin{abstract}
An efficient numerical method called the Preconditioned Bi-Conjugate Gradient (Pre-BiCG) method is presented for the solution of radiative transfer equation in spherical geometry. A variant of this method called Stabilized Preconditioned Bi-Conjugate Gradient (Pre-BiCG-STAB) is also presented. These methods are based on projections on the subspaces of the $n$ dimensional Euclidean space $\mathbb{R}^{n}$ called Krylov subspaces. The methods are shown to be faster in terms of convergence rate compared to the contemporary iterative methods such as Jacobi, Gauss-Seidel and Successive Over Relaxation (SOR).
\end{abstract}

\section{The solar continuum intensity distribution}

\author{
S. Wedemeyer-Böhm ${ }^{1,2}$ and L. Rouppe van der Voort $^{1}$ \\ ${ }^{1}$ Institute of Theoretical Astrophysics, University of Oslo, P.O. Box 1029 Blindern, N-0315 \\ Oslo, Norway \\ ${ }^{2}$ Center of Mathematics for Applications (CMA), University of Oslo, Box 1053 Blindern, \\ N0316 Oslo, Norway
}


Abstract. For many years, there seemed to be significant differences between the continuum intensity distributions derived from observations and simulations of the solar photosphere. In order to settle the discussion on these apparent discrepancies, we present a detailed comparison between simulations and seeing-free observations that takes into account the crucial influence of instrumental image degradation. We use a set of images of quiet Sun granulation taken in the blue, green and red continuum bands of the Broadband Filter Imager of the Solar Optical Telescope (SOT) onboard Hinode. The images are deconvolved with Point Spread Functions (PSF) that account for non-ideal contributions due to instrumental stray-light and imperfections. In addition, synthetic intensity images are degraded with the corresponding PSFs. The results are compared with respect to spatial power spectra, intensity histograms, and the centreto-limb variation of the intensity contrast. The observational findings are well matched with corresponding synthetic observables from three-dimensional radiation (magneto-)hydrodynamic simulations. We conclude that the intensity contrast of the solar continuum intensity is higher than usually derived from ground-based observations and is well reproduced by modern numerical simulations. Properly accounting for image degradation effects is of crucial importance for comparisons between observations and numerical models. It finally settles the traditionally perceived conflict between observations and simulations.

\title{
Temperature stratification in the Sun's photosphere in high horizontal resolution using Ca II $\mathrm{H}$ filtergrams
}

\author{
V. M. J. Henriques ${ }^{1,2}$ and D. Kiselman ${ }^{1}$ \\ ${ }^{1}$ Institute for Solar Physics, Royal Swedish Academy of Sciences, AlbaNova University Center, \\ SE-106 91 Stockholm, Sweden \\ ${ }^{2}$ Stockholm Observatory, Stockholm University, Sweden
}

Abstract. A method to extract the temperature stratification in the Sun's photosphere using filtergrams is presented along with some high resolution results. The data was acquired with the Swedish 1-m Solar Telescope (SST) using a tunable filter in the Ca II H blue wing. Each full scan is completed in the order of seconds thus allowing for the full resolution of the SST and reasonable depth sampling to be obtained simultaneously in a shorter time than that of the evolution time scale of the photosphere. We test the quality of the method by applying it to a set of synthetic images (obtained through radiative transfer on 3D HD and MHD simulation snapshots followed by degradation) and comparing the output with the known 3D simulated atmosphere. Fine structure around bright points becomes evident in both the temperature gradient maps computed from a set of test observations and synthetic images obtained from MHD simulations.

\section{Solar abundances and granulation effects}

\author{
E. Caffau ${ }^{1}$, H.-G. Ludwig ${ }^{2,1}$ and M. Steffen ${ }^{3}$ \\ ${ }^{1}$ GEPI, Observatoire de Paris, CNRS, Université Paris Diderot, Place Jules Janssen, \\ 92190 Meudon, France \\ ${ }^{2}$ CIFIST Marie Curie Excellence Team \\ ${ }^{3}$ Astrophysikalisches Institut Potsdam, An der Sternwarte 16, D-14482 Potsdam, Germany
}

Abstract. The solar abundances have undergone a major downward revision in the last decade, reputedly as a result of employing 3D hydrodynamical simulations to model the inhomogeneous structure of the solar photosphere. The very low oxygen abundance advocated by Asplund et al. (2004), $\mathrm{A}(\mathrm{O})=8.66$, together with the downward revision of the carbon and nitrogen abundances, has created serious problems for solar models to explain the helioseismic measurements.

In an effort to contribute to the dispute we have re-derived photospheric abundances of several elements independently of previous analysis. We applied a state-of-the art 3D (CO5BOLD) 
hydrodynamical simulation of the solar granulation as well as different $1 \mathrm{D}$ model atmospheres for the line by line spectroscopic abundance determinations. The analysis is based on both standard disc-centre and disc-integrated spectral atlases; for oxygen we acquired in addition spectra at different heliocentric angles. The derived abundances are the result of equivalent width and/or line profile fitting of the available atomic lines. We discuss the different granulation effects on solar abundances and compare our results with previous investigations. According to our investigations hydrodynamical models are important in the solar abundance determination, but are not responsible for the recent downward revision in the literature of the solar metallicity.

\title{
Computation and analysis of gyrosyncrothron emission in solar flares
}

\author{
T. S. N. Pinto and J. E. R. Costa \\ Instituto Nacional de Pesquisas Espaciais - Divisão de Astrofísica, Av. dos Astronautas, 1758, \\ CEP 12227-010, São José dos Campos - SP, Brazil
}

\begin{abstract}
The emission spectrum of solar flares in the range of microwave wavelengths is known to be due to the gyrosynchrotron mechanism. In this work, this emission for a few solar flares of the cycle 23 observed by the Nobeyama Radio Observatory instruments were analyzed. The information provided by the time profiles and the event images were used as the input parameters in the numerical computation of two flares' spectrum. A priori information obtained from the data was the electron spectral index, the emitting area and the angle between the magnetic field lines and the observer's line of sight. With these in hands, and following Dulk (1985) and Costa (2005) the first guesses of the magnetic field intensities were obtained. Based on a homogeneous source model where the depth is considered to be a fraction of the observed width, the emission spectra were fitted with the $\chi^{2}$ criterion, with the non-thermal electron number density and the magnetic induction set as free parameters. With this simplified scenario the values obtained for one of the events were the typical, as can be seen from other flares' analysis in the literature. This flare showed an emission peak at $17 \mathrm{GHz}$ resulting in an inferred magnetic induction of $663 \mathrm{G}$ and an electron density of $2.6 \times 10^{6} \mathrm{~cm}^{-3}$. For the second flare, the fitted spectrum showed many harmonics in frequencies higher than the peak emission $(9 \mathrm{GHz})$ that resulted in a very high magnetic induction $(2183 \mathrm{G})$ and a very low electron density $\left(3.8 \times 10^{5} \mathrm{~cm}^{-3}\right)$. This analysis was a first step to model a sample of selected flares using anisotropic magnetic fields extrapolated from the photosphere using the force-free hypothesis. The final analysis will be done solving the transfer equation in this anisotropic ambient.
\end{abstract}

\section{Testing 3D solar models against observations}

\author{
T. M. D. Pereira ${ }^{1,2}$, M. Asplund ${ }^{3}$ and D. Kiselman ${ }^{2}$ \\ ${ }^{1}$ Research School of Astronomy and Astrophysics, Australian National University, Cotter Rd., \\ Weston, ACT 2611, Australia \\ ${ }^{2}$ The Institute for Solar Physics of the Royal Swedish Academy of Sciences, AlbaNova \\ University Center, 10691 Stockholm, Sweden \\ ${ }^{3}$ Max-Planck-Institut für Astrophysik, Postfach 1317, D-85741 Garching b. München, \\ Germany
}

Abstract. We present results from a series of observational tests to 3D and 1D solar models. In particular, emphasis is given to the line formation of atomic oxygen lines, used to derive the much debated solar oxygen photospheric abundance. Using high-quality observations obtained with the Swedish Solar Telescope (SST) we study the center-to-limb variation of the O I lines, testing the models and line formation (LTE and non-LTE). For the O I $777 \mathrm{~nm}$ triplet, the center-to-limb variation sets strong constraints in the non-LTE line formation, and is used to derive an empirical correction factor $\left(S_{\mathrm{H}}\right)$ to the classical Drawin recipe for neutral hydrogen 
collisions. Taking advantage of the spatially-resolved character of the SST data, an additional framework for testing the 3D model and line formation is also studied. From the tests we confirm that the employed 3D model is realistic and its predictions agree very well with the observations.

\title{
Doppler shifts in the transition region and corona
}

\author{
P. Zacharias ${ }^{1}$, S. Bingert ${ }^{1}$ and H. Peter ${ }^{2}$ \\ ${ }^{1}$ Kiepenheuer Institut für Sonnenphysik - Schöneckstrasse 6, D-79104 Freiburg, Germany \\ ${ }^{2}$ Max-Planck-Institut für Sonnensystemforschung - Max-Planck-Strasse 2, D-37191 \\ Katlenburg-Lindau, Germany
}

\begin{abstract}
Emission lines in the transition region and corona show persistent line shifts. It is a major challenge to understand the dynamics in the upper atmosphere and thus these line shifts, which are a signature of the mass cycle between the chromosphere and the corona. We examine EUV emission line profiles synthesized from a 3D MHD coronal model of a solar-like corona, in particular of an active region surrounded by strong chromospheric network. This allows us to investigate the physical processes leading to the line Doppler shifts, since we have access to both, the synthetic spectra and the physical parameters, i.e. magnetic field, temperature and density in the simulation box. By analyzing the evolution of the flows along field lines together with the changing magnetic structure we can investigate the mass cycle. We find evidence that loops are loaded with mass during a reconnection process, leading to upflows. After the loops disconnect from the reconnection site, they cool and drain which leads to the observed redshifts. Previous 1D loop models (neglecting the 3D nature) assumed that heating leads to evaporation and upflows followed by a cooling phase after the heating stops. The scenario modeled here is quite different, as it shows that the continuously changing three-dimensional magnetic structure is of pivotal importance to understand the mass balance between the chromosphere and the corona.
\end{abstract}

\section{Cloud formation and dynamics in cool dwarf and hot exoplanetary atmospheres}

\author{
Adam J. Burgasser \\ Center of Astrophysics and Space Sciences, University of California, 9500 Gilman Dr., San \\ Diego, CA 92093, USA
}

Abstract. The lowest-mass stars, brown dwarfs and extrasolar planets present challenges and opportunities for understanding dynamics and cloud formation processes in low-temperature atmospheres. For brown dwarfs, the formation, variation and rapid depletion of photospheric clouds in L- and T-type dwarfs, and spectroscopic evidence for non-equilibrium chemistry associated with vertical mixing, all point to a fundamental role for dynamics in vertical abundance distributions and cloud/grain formation cycles. For exoplanets, azimuthal heat variations and the detection of stratospheric and exospheric layers indicate multi-layered, asymmetric atmospheres that may also be time-variable (particularly for systems with highly elliptical orbits). Dust and clouds may also play an important role in the thermal energy balance of exoplanets through albedo effects. For all of these cases, 3D atmosphere models are becoming an increasingly essential tool for understanding spectral and temporal properties. In this review, I summarize the observational evidence for clouds and dynamics in cool dwarf and hot exoplanetary atmospheres, outstanding problems associated with these processes, and areas where effective synergy can be achieved. 


\title{
Reflectance spectra of earth-like exoplanets
}

M. Wagner and P. H. Hauschildt

Hamburger Sternwarte, Gojenbergsweg 112, 21029 Hamburg

Abstract. Numerical simulations on irradiated exoplanets provide spectra that contain informations about the temperature- and density structure and chemical composition of the exoplanetary atmosphere. The calculation of cool objects is challenging, because of the much more complex chemistry, i.e. the strong molecular abundances as well as the occurrence of dust formation. In order to create an object with planetary features, such as size, temperature and abundances, the stellar atmosphere code PHOENIX had to be adapted in an appropriate way. Starting with an object of Venus-like parameters in 1D (spherical setup), temperature and optical depth will be reduced to Earth-like values. But in an optically thin atmosphere, what influence might the surface texture have on the combined spectrum? An albedo module has already been embedded to serve the cases of non-angular dependence (e.g. soils, vegetation) and angular dependence, i.e. water surface. The aim is to expand the work to $3 \mathrm{D}$.

\section{Simulations of dust clouds in the atmospheres of substellar objects}

\author{
B. Freytag ${ }^{1,3}$, F. Allard ${ }^{1,2}$, H.-G. Ludwig ${ }^{3}$, D. Homeier ${ }^{4}$ and M. Steffen ${ }^{5}$ \\ ${ }^{1}$ Centre de Recherche Astrophysique de Lyon, UMR 5574: CNRS, Université de Lyon, École \\ Normale Supérieure de Lyon, 46 allée d'Italie, F-69364 Lyon Cedex 07, France \\ ${ }^{2}$ Institut dÁstrophysique de Paris, UMR 7095: CNRS, Université Pierre et Marie Curie-Paris \\ 6, 98bis boulevard Arago, 75014 Paris, France \\ ${ }^{3}$ Observatoire de Paris-Meudon, GEPI-CIFIST, F-92195 Meudon, France \\ ${ }^{4}$ Institut für Astrophysik Göttingen, Georg-August-Universität, Friedrich-Hund-Platz 1, \\ D-37077 Göttingen, Germany \\ ${ }^{5}$ Astrophysikalisches Institut Potsdam, An der Sternwarte 16, D-14482 Potsdam, Germany
}

Abstract. The atmospheres of brown dwarfs allow the formation of dust grains and their rainout into deeper, invisible layers. However, observed spectra of L dwarfs can only be reproduced when static 1D models account for dust formation and its resulting greenhouse effect in the visible layers. Time-dependent hydrodynamical processes can mix up the material giving rise to complex unsteady weather phenomena on these objects. We performed radiation hydrodynamics simulations in two and three dimensions of the atmospheres of brown dwarfs with CO5BOLD, including a treatment of dust particles. We find that exponential overshoot (close to the gas convection zone), gravity waves (weak omni-present mixing), and convection within dust layers (in the thick clouds in cooler models) contribute to the atmospheric mixing, which is far from being a stationary process. The presence of dust in the atmospheres is accompanied by large temporal and spatial intensity fluctuations.

\section{Brown dwarf parallax programs}

\author{
R. L. Smart \\ Istituto Nazionale di Astrofisica - Osservatorio Astronomico di Torino, Strada \\ Osservatorio 20, 10025 Pino Torinese, Italy
}

Abstract. Parallaxes are crucial for many brown dwarf topics from the substellar mass function to $3 \mathrm{D}$ atmospheric modeling. Here we discuss the current sample of brown dwarfs with parallaxes and the prospects for the near future. 


\title{
Polarization : Proving ground for methods in radiative transfer
}

\author{
K. N. Nagendra ${ }^{1}$, L. S. Anusha ${ }^{1}$ and M. Sampoorna ${ }^{2}$ \\ ${ }^{1}$ Indian Institute of Astrophysics, Koramangala, Bangalore 560 034, India \\ ${ }^{2}$ Instituto de Astrofisica de Canarias, E-38205, La Laguna, Tenerife, Spain
}

Abstract. Polarization of solar lines arises due to illumination of radiating atom by anisotropic (limb darkened/brightened) radiation. Modelling the polarized spectra of the Sun and stars requires solution of the line radiative transfer problem in which the relevant polarizing physical mechanisms are incorporated. The purpose of this paper is to describe in what different ways the polarization state of the radiation 'complicates' the numerical methods originally designed for scalar radiative transfer. We present several interesting situations involving the solution of polarized line transfer to prove our point. They are (i) Comparison of the polarized approximate lambda iteration (PALI) methods with new approaches like Bi-conjugate gradient method that is faster, (ii) Polarized Hanle scattering line radiative transfer in random magnetic fields, (iii) Difficulties encountered in incorporating polarized partial frequency redistribution (PRD) matrices in line radiative transfer codes, (iv) Technical difficulties encountered in handling polarized specific intensity vector, some components of which are sign changing, (v) Proving that scattering polarization is indeed a boundary layer phenomenon. We provide credible benchmarks in each of the above studies. We show that any new numerical methods can be tested in the best possible way, when it is extended to include polarization state of the radiation field in line scattering.

\section{Magnetic structuring at spatially unresolved scales}

\author{
J. O. Stenflo ${ }^{1,2}$ \\ ${ }^{1}$ Institute of Astronomy, ETH Zurich, HIT J 23.6, CH-8093 Zurich \\ ${ }^{2}$ Istituto Ricerche Solari Locarno (IRSOL), Via Patocchi, CH-6605 Locarno-Monti
}

Abstract. Magneto-convection structures the Sun's magnetic field down to the magnetic diffusion scale of order $10 \mathrm{~m}$, where the field ceases to be frozen-in. This is about four orders of magnitude below the current resolution limit of solar telescopes. The subpixel structuring has a dramatic effect on the derived, spatially averaged flux densities in the resolved domain, in particular on the angular distribution of the field. Thus we find that the previously reported apparent predominance of horizontal magnetic flux on the quiet Sun is an artefact of the subresolution structuring. Here we try to clarify how Stokes profile data may be used to explore the spatially unresolved domain. Insert text of abstract

\section{Modeling the second solar spectrum}

\author{
M. Sampoorna \\ Instituto de Astrofísica de Canarias, E-38205 La Laguna, Tenerife, Spain.
}

\begin{abstract}
We present an empirical approach to model the wing polarization of strong resonance lines. This procedure based on 'last scattering approximation' (LSA) was developed by Stenflo (1980) and Stenflo (1982), for coherent scattering in the laboratory frame. We generalize his empirical approach to handle partial frequency redistribution (PRD). We illustrate this approach by applying it to the Ca I $4227 \AA$ line. The LSA approach is successful in reproducing the observed Stokes $Q / I$ polarization, including the location of the wing polarization maxima and the minima around the Doppler core, but fails to reproduce the observed spatial variations of the far wing polarization in terms of magnetic field and PRD effects. This null result points in the direction of a non-magnetic origin, which may include local deviations from a plane-parallel stratification with an inhomogeneous solar atmosphere.
\end{abstract}




\title{
3D stellar atmospheres for stellar structure models and asteroseismology
}

\begin{abstract}
F. Kupka ${ }^{1,2}$
${ }^{1}$ Observatoire de Paris, LESIA, CNRS UMR 8109, F-92195 Meudon, France

${ }^{2}$ Institute of Mathematics, University of Vienna, Nordbergstraße 15, A-1090 Vienna, Austria

Abstract. Convection is the most important physical process that determines the structure of the envelopes of cool stars. It influences the surface radiation flux and the shape of observed spectral line profiles and is responsible for both generating and damping solar-like oscillations, among others. 3D numerical simulations of stellar surface convection have developed into a powerful tool to model and analyse the physical mechanisms operating at the surface of cool stars. This review discusses the main principles of 3D stellar atmospheres used for such applications. The requirements from stellar structure and evolution theory to use them as boundary conditions are analysed as well as the capabilities of using helio- and asteroseismology to reduce modelling uncertainties and probing the consistency and accuracy of 3D stellar atmospheres as part of this process. Simulations for the solar surface made by different teams are compared and some issues concerning the uncertainties of this modelling approach are discussed.
\end{abstract}

\section{The CIFIST 3D model atmosphere grid}

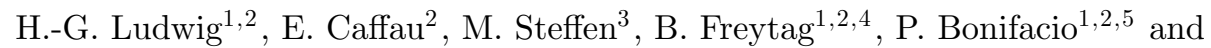
A. Kučinskas ${ }^{6,7}$

${ }^{1}$ CIFIST - Marie Curie Excellence Team

${ }^{2}$ GEPI - Observatoire de Paris, CNRS, Université Paris Diderot, 92195 Meudon, France

${ }^{3}$ Astrophysikalisches Institut Potsdam, An der Sternwarte 16, 14482 Potsdam, Germany

${ }^{4}$ CRAL - UMR 5574 CNRS, Université de Lyon, École Normale Supérieure de Lyon, 46 allée d'Italie, 69364 Lyon Cedex 07, France

${ }^{5}$ INAF - Osservatorio Astronomico di Trieste, via Tiepolo 11, 34143 Trieste, Italy ${ }^{6}$ Institute of Theoretical Physics and Astronomy, Goštauto 12, Vilnius LT-01108, Lithuania

${ }^{7}$ Vilnius University Astronomical Observatory, Ciurlionio 29, Vilnius LT-03100, Lithuania

Abstract. Grids of stellar atmosphere models and associated synthetic spectra are numerical products which have a large impact in astronomy due to their ubiquitous application in the interpretation of radiation from individual stars and stellar populations. 3D model atmospheres are now on the verge of becoming generally available for a wide range of stellar atmospheric parameters. We report on efforts to develop a grid of 3D model atmospheres for late-type stars within the CIFIST Team at Paris Observatory. The substantial demands in computational and human labor for the model production and post-processing render this apparently mundane task a challenging logistic exercise. At the moment the CIFIST grid comprises 77 3D model atmospheres with emphasis on dwarfs of solar and sub-solar metallicities. While the model production is still ongoing, first applications are already worked upon by the CIFIST Team and collaborators.

\section{Spatially resolving the inhomogeneous structure of the dynamical atmosphere of Betelgeuse with VLTI/AMBER}

\author{
K. Ohnaka \\ Max-Planck-Institut für Radioastronomie, Auf dem Hügel 69, 53121 Bonn, Germany
}


Abstract. We present spatially resolved high-spectral resolution $K$-band observations of the red supergiant Betelgeuse ( $\alpha$ Ori) using AMBER at the Very Large Telescope Interferometer (VLTI). IR long-baseline interferometry combined with spectral resolutions of 4800-12000 enables us to probe the inhomogeneous structures in the dynamical atmosphere of Betelgeuse using the CO first overtone lines near $2.3 \mu \mathrm{m}$. Our AMBER observations mark the highest spatial resolution (9 mas) achieved for Betelgeuse with five resolution elements over its stellar disk. The AMBER data in the CO lines reveal salient inhomogeneous structures. Particularly, the visibilities and differential/closure phases within the $\mathrm{CO}$ lines show that the blue and red wings of the lines originate in spatially distinct regions over the stellar disk, clearly demonstrating an inhomogeneous velocity field in the atmosphere of Betelgeuse. The AMBER observations in the $\mathrm{CO}$ lines can be roughly explained by a simple model, in which a patch of $\mathrm{CO}$ gas is moving outward or inward with velocities of $10-15 \mathrm{~km} \mathrm{~s}^{-1}$, while the $\mathrm{CO}$ gas in the remaining region in the atmosphere is moving in the opposite direction at the same velocities. This model also suggests the presence of dense molecular layers extending to $\sim 1.4-1.5 R_{\star}$ with a CO column density of $\sim 1 \times 10^{20} \mathrm{~cm}^{-2}$. Our AMBER observations of Betelgeuse in the CO first overtone lines are the first spatially resolved study of the gas motion in a stellar atmosphere (photosphere and extended, warm molecular layers) other than the Sun and have opened a door to a better understanding of macroturbulence.

\title{
Abundance analysis of the halo giant HD 122563 with three-dimensional model stellar atmospheres
}

\author{
R. Collet ${ }^{1}$, A. Nordlund ${ }^{2}$, M. Asplund ${ }^{1}$, W. Hayek ${ }^{3,1}$ and R. Trampedach ${ }^{3}$ \\ ${ }^{1}$ Max-Planck-Institut für Astrophysik, Postfach 1317, D-85741 Garching b. München \\ ${ }^{2}$ Niels Bohr Institute, University of Copenhagen, Juliane Maries Vej 30, DK-2100, \\ Copenhagen, Denmark \\ ${ }^{3}$ Research School of Astronomy and Astrophysics, Mount Stromlo Observatory, Cotter Road, \\ Weston ACT 2611, Australia
}

Abstract. We present a preliminary local thermodynamic equilibrium (LTE) abundance analysis of the template halo red giant HD122563 based on a realistic, three-dimensional (3D), time-dependent, hydrodynamical model atmosphere of the very metal-poor star. We compare the results of the $3 \mathrm{D}$ analysis with the abundances derived by means of a standard LTE analysis based on a classical, 1D, hydrostatic model atmosphere of the star. Due to the different upper photospheric temperature stratifications predicted by $1 \mathrm{D}$ and $3 \mathrm{D}$ models, we find large, negative, 3D-1D LTE abundance differences for low-excitation $\mathrm{OH}$ and Fe I lines. We also find trends with lower excitation potential in the derived Fe LTE abundances from Fe I lines, in both the $1 \mathrm{D}$ and $3 \mathrm{D}$ analyses. Such trends may be attributed to the neglected departures from LTE in the spectral line formation calculations.

\section{D hydrodynamical simulations of stellar photospheres with the $\mathrm{CO}^{5} \mathrm{BOLD}$ code}

\author{
A. Kučinskas ${ }^{1,2}$, H.-G. Ludwig ${ }^{3}$, E. Caffau ${ }^{3}$ and M. Steffen ${ }^{4}$ \\ ${ }^{1}$ Institute of Theoretical Physics and Astronomy, Goštauto 12, Vilnius LT-01108, Lithuania \\ ${ }^{2}$ Vilnius University Astronomical Observatory, Ciurlionio 29, Vilnius LT-03100, Lithuania \\ ${ }^{3}$ GEPI - CIFIST, Observatoire de Paris-Meudon, 5 place Jules Janssen, 92195 Meudon Cedex, \\ France \\ ${ }^{4}$ Astrophysikalisches Institut Potsdam, An der Sternwarte 16, D-14482 Potsdam, Germany \\ Abstract. We present synthetic broad-band photometric colors of a late-type giant located close \\ to the RGB tip $\left(T_{\text {eff }} \approx 3640 \mathrm{~K}, \log g=1.0\right.$ and $\left.[\mathrm{M} / \mathrm{H}]=0.0\right)$. Johnson-Cousins-Glass BVRIJHK
}


colors were obtained from the spectral energy distributions calculated using 3D hydrodynamical and $1 \mathrm{D}$ classical stellar atmosphere models. The differences between photometric magnitudes and colors predicted by the two types of models are significant, especially at optical wavelengths where they may reach, e.g., $\Delta V \approx 0.16, \Delta R \approx 0.13$ and $\Delta(V-I) \approx 0.14, \Delta(V-K) \approx 0.20$. Differences in the near-infrared are smaller but still non-negligible (e.g., $\Delta K \approx 0.04$ ). Such discrepancies may lead to noticeably different photometric parameters when these are inferred from photometry (e.g., effective temperature will change by $\Delta T_{\text {eff }} \approx 60 \mathrm{~K}$ due to difference of $\Delta(V-K) \approx 0.20)$.

\title{
The effective temperature scale: resolving different versions
}

L. Casagrande

Max Planck Institute for Astrophysics, Postfach 1317, 85741 Garching, Germany

Abstract. The effective temperature of a stellar surface is a measure of the total energy and its correct characterization plays a central role in both theory and observations. Various effective temperature scales have been proposed in literature. Despite being such a long-lived tradition and the high internal precision usually achieved, systematic differences of order $100 \mathrm{~K}$ among various scales are still present, thus hindering much of a progress in the field. We present an Infrared Flux Method based investigation aimed to carefully assess the sources of such discrepancies and pin down their origin. We break the impasse among different scales by using a large set of solar twins, stars which are spectroscopically and photometrically identical to the Sun, to set the zero-point of the effective temperature scale to within few degrees. Our newly calibrated, precise and accurate temperature scale applies to dwarfs and subgiants, from super solar metallicities to the most metal poor stars currently known. The effect of using spectral energy distribution computed from 3D models in the Infrared Flux Method, as well as 3D synthetic colours are also briefly outlined.

\section{Micro- and macroturbulence derived from 3D hydrodynamical stellar atmospheres}

\author{
M. Steffen ${ }^{1}$, H.-G. Ludwig ${ }^{2,3}$ and E. Caffau ${ }^{3}$ \\ ${ }^{1}$ Astrophysikalisches Institut Potsdam, An der Sternwarte 16, D-14482 Potsdam, Germany \\ ${ }^{2}$ CIFIST Marie Curie Excellence Team \\ ${ }^{3}$ GEPI - Observatoire de Paris, CNRS, Université Paris Diderot; 92195 Meudon, France
}

Abstract. The theoretical prediction of micro- and macroturbulence $\left(\xi_{\mathrm{mic}}\right.$ and $\left.\xi_{\mathrm{mac}}\right)$ as a function of stellar parameters can be useful for spectroscopic work based on 1D model atmospheres in cases where an empirical determination of $\xi_{\text {mic }}$ is impossible due to a lack of suitable lines and/or macroturbulence and rotational line broadening are difficult to separate. In an effort to exploit the CIFIST 3D model atmosphere grid for deriving the theoretical dependence of $\xi_{\mathrm{mic}}$ and $\xi_{\mathrm{mac}}$ on effective temperature, gravity, and metallicity, we discuss different methods to derive $\xi_{\mathrm{mic}}$ from the numerical simulations, and report first results for the Sun and Procyon. In both cases the preliminary analysis indicates that the microturbulence found in the simulations is significantly lower than in the real stellar atmospheres.

\section{D molecular line formation in dwarf carbon-enhanced metal-poor stars}




\author{
N. T. Behara ${ }^{1,2}$, H.-G. Ludwig ${ }^{1,2}$, P. Bonifacio ${ }^{1,2,3}$, L. Sbordone ${ }^{1,2}$, \\ J. I. González Hernández ${ }^{1,2}$ and E. Caffau ${ }^{2}$ \\ ${ }^{1}$ CIFIST Marie Curie Excellence Team \\ ${ }^{2}$ GEPI, Observatoire de Paris CNRS, Université Paris Diderot, Place Jules Janssen, \\ 92190 Meudon, France \\ ${ }^{3}$ Istituto Nazionale di Astrofisica - Osservatorio Astronomico di Trieste Via Tiepolo 11, \\ I-34143 Trieste, Italy
}

Abstract. We present a detailed analysis of the carbon and nitrogen abundances of two dwarf carbon-enhanced metal-poor (CEMP) stars: SDSS J1349-0229 and SDSS J0912+0216. We also report the oxygen abundance of SDSS J1349-0229. These stars are metal-poor, with $[\mathrm{Fe} / \mathrm{H}]<$ -2.5 , and were selected from our ongoing survey of extremely metal-poor dwarf candidates from the Sloan Digital Sky Survey (SDSS). The carbon, nitrogen and oxygen abundances rely on molecular lines which form in the outer layers of the stellar atmosphere. It is known that convection in metal-poor stars induces very low temperatures which are not predicted by 'classical' 1D stellar atmospheres. To obtain the correct temperature structure, one needs full 3D hydrodynamical models. Using CO5BOLD 3D hydrodynamical model atmospheres and the Linfor3D line formation code, molecular lines of $\mathrm{CH}, \mathrm{NH}, \mathrm{OH}$ and $\mathrm{C}_{2}$ were computed, and 3D carbon, nitrogen and oxygen abundances were determined. The resulting carbon abundances were compared to abundances derived using atomic C I lines in 1D LTE and NLTE. For one star, SDSS J1349-0229, we were able to compare the 3D oxygen abundance from $\mathrm{OH}$ lines to $\mathrm{O}$ I lines in 1D LTE and NLTE. There is not a good agreement between the carbon abundances determined from $\mathrm{C}_{2}$ bands and from the $\mathrm{CH}$ band, and molecular lines do not agree with the atomic $\mathrm{C}$ I lines. Although this may be partly due to uncertainties in the transition probabilities of the molecular bands it certainly has to do with the temperature structure of the outer layers of the adopted model atmosphere. In fact the discrepancy between $\mathrm{C}_{2}$ and $\mathrm{CH}$ is in opposite directions when using 3D and 1D models. Confronted with this inconsistency, we explore the influence of the $3 \mathrm{D}$ model properties on the molecular abundance determination. In particular, the choice of the number of opacity bins used in the model calculations and its subsequent effects on the temperature structure and molecular line formation is discussed.

\title{
Effects of granulation on neutral copper resonance lines in metal-poor stars
}

\author{
P. Bonifacio ${ }^{1,2,3}$, E. Caffau ${ }^{2}$ and H.-G. Ludwig ${ }^{1,2}$ \\ ${ }^{1}$ CIFIST Marie Curie Excellence Team \\ ${ }^{2}$ GEPI, Observatoire de Paris, CNRS, Université Paris Diderot; Place Jules Janssen, \\ 92190 Meudon, France \\ ${ }^{3}$ Istituto Nazionale di Astrofisica - Osservatorio Astronomico di Trieste, Via Tiepolo 11, \\ I-34143 Trieste, Italy
}

Abstract. We make use of three dimensional hydrodynamical simulations to investigate the effects of granulation on the $\mathrm{Cu}$ I lines of Mult. 1 in the near UV, at $324.7 \mathrm{~nm}$ and $327.3 \mathrm{~nm}$. These lines remain strong even at very low metallicity and provide the opportunity to study the chemical evolution of $\mathrm{Cu}$ in the metal-poor populations. We find very strong granulation effects on these lines. In terms of abundances the neglect of such effects can lead to an overestimate of the $\mathrm{A}(\mathrm{Cu})$ by as much as 0.8 dex in dwarf stars. Comparison of our computations with stars in the metal-poor Globular Clusters NGC 6752 and NGC 6397, show that there is a systematic discrepancy between the copper abundances derived from Mult. 2 in TO stars and those derived in giant stars of the same cluster from the lines of Mult. 2 at at $510.5 \mathrm{~nm}$ and $587.2 \mathrm{~nm}$. We conclude that the $\mathrm{Cu}$ I resonance lines are not reliable indicators of $\mathrm{Cu}$ abundance and we believe that an investigations of departures from LTE is mandatory to make use of these lines. 


\title{
Monitoring mass motions of Betelgeuse's photosphere using robotic telescopes
}

\author{
M. Weber, T. Carroll, T. Granzer, M. Steffen and K. G. Strassmeier \\ Astrophysikalisches Institut Potsdam, An der Sternwarte 16, D-14482 Potsdam, Germany
} Abstract. We started monitoring Betelgeuse using STELLA/SES, the STELLA échelle spectrograph fed by a robotic $1.2 \mathrm{~m}$ telescope on Tenerife, and the automatic photometric telescope (APT) T7 in Arizona in fall 2008. In this first observing season, we have collected 67 high resolution spectra from 390 to $900 \mathrm{~nm}$ at a resolution of 50,000 and a S/N between 100 and 300 , and a comparable number of photometric observations in the $\mathrm{H} \alpha$ filter. In this presentation, we report on the initial findings based on this first data set: Radial velocities, effective temperature (along with surface gravity and metallicity) are automatically computed by the STELLA/SES data reduction \& analysis pipeline. We compare these global measurements and the photometric brightness with velocities and temperature indicators derived from individual spectral lines, to bring these values in line with recently published observations. Furthermore we compute synthetic line profiles from state-of-the-art 3D stellar convection models, and compare the line-profiles, their shapes and positions to our observations. The final aim of the observing program is to find out if the spectral line variations can be explained using these non-magnetic convection models.

\section{References}

Asplund, M., Grevesse, N., Sauval, A. J., Allende Prieto, C., \& Kiselman, D. 2004, A\&A 417, 751

Costa, J. E. R. 2005, in: Proceedings of Magnetic Fields in the Universe, 544

Dulk, G. A. 1985, ARAA 23, 169

Stenflo, J. O. 1980, A\&̈A 84, 68

Stenflo, J. O. 1982, Solar Phys. 80, 209 